\title{
SEMI-SIMPLE EXTENSIONS OF RINGS
}

\author{
OSCAR GOLDMAN
}

In this paper we investigate the conditions under which a given ring is a sub-ring of a semi-simple ring. ${ }^{1}$ For convenience, we say that a ring $A$ is an extension of a ring $B$, if $B$ is a sub-ring of $A$. It is found that the existence of a semi-simple extension is equivalent to the vanishing of the extension radical, a two-sided ideal defined analogously to the ordinary radical. In Theorem II we give an intrinsic characterization of the extension radical, where we find that the latter is determined by the addition in the ring and is independent of the multiplication. This result is summarized in Theorem III.

For the convenience of the reader, we reproduce here some of the definitions given in the paper mentioned in footnote 1 . The radical of a ring $A$ is obtained as the intersection of the annihilators of all simple $A$-modules. When the radical consists only of the zero element of $A$, we say that the ring is semi-simple. The radical as defined here contains the ideal classically known as the radical (the sum of all nilpotent left ideals) and is equal to it if one assumes tht $A$ satisfies the descending chain condition on left ideals. A ring which is semisimple in the present sense has then no nilpotent ideals.

In order to define the extension radical, we must first introduce the auxiliary notion of a quasi-simple module. If $\mathfrak{M}$ is an abelian group, denote by $E(\mathfrak{M})$ the ring of all endomorphisms of $\mathfrak{M}$. We say that $\mathfrak{M}$ is a quasi-simple group if $\mathfrak{M}$ is a simple $E(\mathfrak{M})$-module. An $A$-module $\mathfrak{M}$ is a quasi-simple module if the underlying additive group of $\mathfrak{M}$ is quasi-simple. We shall find the following two lemmas useful.

Lemma I. If $\mathfrak{M}$ is a simple A-module, then $\mathfrak{M}$ is a quasi-simple module.

Let $\mathfrak{A}$ denote the annihilator of $\mathfrak{M}$. It is clear that $\mathfrak{M}$ is a simple $A / \mathfrak{A}$-module, and furthermore that $A / \mathfrak{A} \subset E(\mathfrak{M})$. Thus $\mathfrak{M}$ is certainly simple for $E(\mathfrak{M})$.

LEMMA II. Every quasi-simple group is the underlying additive group of a vector space over a field and conversely.

Let $Z$ be the center of $E(\mathfrak{M})$. Since $\mathfrak{M}$ is a simple $E(\mathfrak{M})$-module, $Z$ is

Received by the editors June 6, 1946.

${ }^{1}$ For the definitions and elementary properties of the concepts involved in the study of semi-simple rings, see my previous paper, $A$ characterization of semi-simple rings, Bull. Amer. Math. Soc. vol. 52 (1946) pp. 1021-1027. 
a field by Schur's lemma, and $\mathfrak{M}$ can be considered as a vector space over $Z$. (The precise form of Schur's lemma used here is given in the reference cited in footnote 1 .)

If $\mathfrak{M}$ is a vector space over a field $K$, let $E^{\prime}$ be the ring of all linear transformations of $\mathfrak{M}$. It can easily be seen ${ }^{2}$ that $\mathfrak{M}$ is a simple $E^{\prime}$ module; but $E^{\prime} \subset E(\mathfrak{M})$, so that $\mathfrak{M}$ is a simple $E(\mathfrak{M})$-module, and is therefore a quasi-simple group.

By Lemma II we can associate an integer, either zero or a prime, to each quasi-simple group, $\mathfrak{M}$, namely, the characteristic of those fields over which $\mathfrak{M}$ can be considered as a vector space.

The following fact will be found useful: If every element of an additive group $\mathfrak{M}$ has the same order $p$, with $p$ a prime, then $\mathfrak{M}$ is a quasi-simple group since it can be considered as a vector space over the primitive field of characteristic $p$.

We now define the extension radical of a ring $A$ to be the intersection of the annihilators of all quasi-simple $A$-modules. It is clear that the extension radical is a two-sided ideal in $A$. By Lemma $\mathrm{I}$, the extension radical of $A$ is contained in the radical of the ring.

In order to make use of the notion just introduced, it is necessary to review some elementary facts about simple and semi-simple rings. We say that a ring $A$ is simple if there exists a faithful simple $A$-module. From our definitions it is clear that $E(\mathfrak{M})$ is a simple ring if $\mathfrak{M}$ is a quasi-simple group. We call a ring $E(\mathfrak{M})$, where $\mathfrak{M}$ is quasi-simple, a full linear ring. If $\left\{A_{\lambda}\right\}$ is a set of rings, we construct the product of the $A_{\lambda}$ by forming the Cartesian product of the underlying sets (the unrestricted product) and defining the ring operations to be coordinate-wise. (The ring formed in this way is sometimes referred to in the literature as the direct sum of the $A_{\lambda}$.) We have the following lemmas.

LEMMA III. Let $\left\{A_{\lambda}\right\}$ be a set of simple rings, then the product $A$ of the $A_{\lambda}$ is semi-simple.

Let $\mathfrak{M}_{\lambda}$ be a faithful simple $A_{\lambda}$-module. We can consider $\mathfrak{M}_{\lambda}$ as an $A$-module, by writing $\left(\cdots, a_{\lambda}, \cdots, a_{\lambda^{\prime}}, \cdots\right) x=a_{\lambda} x$, for all $x \in \mathfrak{M}_{\lambda}$. As an $A$-module, $\mathfrak{M}_{\lambda}$ is still simple; the annihilator of $\mathfrak{M}_{\lambda}$ is the set of all elements of $A$ whose $\lambda$-coordinate is zero. The intersection of the annihilators of all the modules $\mathfrak{M}_{\lambda}$ considered as $A$-modules is then $\{0\}$, so that $A$ is semi-simple.

${ }^{2}$ The statement made is equivalent to the fact that given a subspace $\mathfrak{R}$ of $\mathfrak{M}$, there exists a basis for $\mathfrak{M}$, a subset of which is a basis for $\mathfrak{R}$. See for example, $S$. Lefschetz, Algebraic topology, Amer. Math. Soc. Colloquium Publications, vol. 27, 1942, p. 73. 
LEMMA IV. Every semi-simple ring is contained in the product of full linear rings.

Let $A$ be a semi-simple ring, $\left\{\mathfrak{M}_{\lambda}\right\}$ a system of simple $A$-modules, the intersection of whose annihilators is $\{0\}$. Since $\mathfrak{M}_{\lambda}$ is a simple $A$-module, $E\left(\mathfrak{M}_{\lambda}\right)$ is a full linear ring, let $E=\Pi_{\lambda} E\left(\mathfrak{M}_{\lambda}\right)$. If $\mathfrak{A}_{\lambda}$ is the annihilator of $\mathfrak{M}_{\lambda}$, we have a homomorphism $\sigma_{\lambda}$ of $A$ into $E\left(\mathfrak{M}_{\lambda}\right)$ whose kernel is $\mathfrak{R}_{\lambda}$. If $\pi_{\lambda}$ is the projection of $E$ onto $E\left(\mathfrak{M}_{\lambda}\right)$, define the homomorphism $\tau$ of $A$ into $E$ by $\pi_{\lambda} \tau=\sigma_{\lambda}$. It is clear that the kernel of $\tau$ is the intersection of the kernels of the $\sigma_{\lambda}$ which we know to be $\{0\}$. Thus $A$ is isomorphically contained in $E_{2}$ the product of full linear rings.

We are now in a position to prove the following theorem.

TheOREM I. Let $A$ be a ring and $\Re$ its extension radical. Then a necessary and sufficient condition that $A$ has a semi-simple extension is that $\Re$ reduces to $\{0\}$.

Suppose that $B$ is a semi-simple extension of $A$; by Lemma IV, $B$ is contained in the product $\prod_{\lambda} E\left(\mathfrak{M}_{\lambda}\right)$ of full linear rings. The $\mathfrak{M}_{\lambda}$ are quasi-simple $A$-modules. By an argument similar to the one used in the proof of Lemma III, the intersection of the annihilators of the $\mathfrak{M}_{\lambda}$ is $\{0\}$, and therefore the extension radical of $A$ reduces to $\{0\}$.

If on the other hand, the extension radical of $A$ is $\{0\}$, let $\left\{\mathfrak{M}_{\lambda}\right\}$ be a system of quasi-simple $A$-modules, the intersection of whose annihilators is $\{0\}$. Defining $E$ to be the product of the $E\left(\mathfrak{M}_{\lambda}\right)$, we know that $E$ is semi-simple. Exactly as in the proof of Lemma IV, we can construct an isomorphism of $A$ into $E$. Thus $A$ has a semi-simple extension.

If $n$ is an integer, define $n A$ to be the set of all elements of $A$ which are of the form $n a$ for some $a \in A$. It is clear that $n A$ is a two-sided ideal in $A$. Denote by $T$ the set of all elements of $A$ which are of finite order in the underlying additive group of $A$. Again $T$ is a two-sided ideal. We have the following theorem.

Theorem II. Let $A$ be a ring, and $\Re$ its extension radical. Then $\Re=T \cap \bigcap_{p} p A$, taken over all prime numbers $p$.

If the characteristic of a quasi-simple $A$-module $\mathfrak{M}$ is $p \neq 0$, it is clear that $p A$ is contained in the annihilator of $\mathfrak{M}$. Again if $\mathfrak{M}$ is of characteristic zero and $a \in T$, so that $n a=0$ for some $n \neq 0$, we have $a x=n a \cdot n^{-1} x=0$ for all $x \in \mathfrak{M}$, so that $T$ is contained in the annihilator of $\mathfrak{M}$. Thus we have $\Re \supset T \cap \cap_{p} p A$.

We now consider the special case in which $A$ has a unit element. 
We shall first show that it is sufficient to consider only quasi-simple $A$-modules in which the unit element acts like the identity automorphism. To be precise, if $\mathfrak{M}$ is a quasi-simple $A$-module, $\mathfrak{A}$ the annihilator of $\mathfrak{M}$, then there is a quasi-simple $A$-module $\mathfrak{N}$, such that the unit element of $A$ acts like the identity in $\mathfrak{N}$, the annihilator of $\mathfrak{N}$ is $\mathfrak{A}$ and the characteristic of $\mathfrak{N}$ is the same as that of $\mathfrak{M}$. Let $\mathfrak{M}_{r}$ be the trivial sub-module of $\mathfrak{M}$, that is, the set of all $x \in \mathfrak{M}$ for which $A x=\{0\}$. If $Z$ is the center of $E(\mathfrak{M}), Z$ is a field, all elements of $Z$ commute with the elements of $A$ modulo $\mathfrak{A}$, so that the elements of $Z$ map $\mathfrak{M}_{T}$ into itself. Then the factor module $\mathfrak{M} / \mathfrak{M}_{T}$ is a quasi-simple $A$-module of the same characteristic as $\mathfrak{M}$. Since, for all $x \in \mathfrak{M}$, $1 x-x \in \mathfrak{M}_{T}$, the unit element of $A$ acts like the identity in $\mathfrak{M} / \mathfrak{M}_{T}$. If $a \in \mathfrak{A}$ it is obvious that $a \mathfrak{M} / \mathfrak{M}_{T}=\{\overline{0}\}$. If $a \mathfrak{M} / \mathfrak{M}_{T}=\{\overline{0}\}$, or $a \mathfrak{M} \subset \mathfrak{M}_{T}$, we have $A a \mathfrak{M}=\{0\}$ or $a \mathfrak{M}=\{0\}$. Thus the annihilator of $\mathfrak{M} / \mathfrak{M}_{T}$ is $\mathfrak{A}$.

If $p$ is a prime, the factor group $A / p A$ has a natural structure as an $A$-module. Furthermore, each element of $A / p A$ has the same prime order so that $A / p A$ is a quasi-simple module. If $a A / p A=\{\overline{0}\}$ or $a A \subset p A$ we have $a \in p A$. Thus the annihilator of $A / p A$ is contained in $p A$ and is therefore equal to $p A$.

If $T=A$, that is, if $n 1=0$, for some $n \neq 0$, all nontrivial quasisimple modules have finite characteristic which divides $n$. For, if $x \in \mathfrak{M}, 1 x=x, n x=n 1 x=0$ so that the characteristic of $\mathfrak{M}$ divides $n$. In that case, by the results of the above paragraph, $\Re \subset T \cap \cap_{p} p A$ and therefore $\Re=T \cap \cap_{p} p A$.

If $T \not A, A / T$ is a nontrivial $A$-module. It is clear that no element of $\mathfrak{M}_{0}=A / T$ has finite order. Consider the tensor product ${ }^{3}$ of the field of rationals $K$ with $\mathfrak{M}_{0}, K \times \mathfrak{M}_{0}$. By the preceding remark, we know that, if $1 \times \bar{a}=0$, then $\bar{a}=\overline{0} . K \times \mathfrak{M}_{0}$ is a vector space over $K$, and has a natural structure as an $A$-module; we define $a^{\prime}(1 \times \bar{a})=1 \times a^{\prime} \bar{a}$, linearity defines the operations of $A$ on all of $K \times \mathfrak{M}_{0}$. If now $a K \times \mathfrak{M}_{0}$ $=\{0\}$, we have in particular, $a(1 \times \overline{1})=1 \times a \overline{1}=0$, or $a \overline{1}=\overline{0}$ whence $a \in T$. Thus the annihilator of $K \times \mathfrak{M}_{0}$ is contained in $T$ and is therefore equal to $T$. We find then again that $\Re \subset T \cap \cap_{p} p A$, so that in the general case, provided $A$ has a unit element, $\Re=T \cap \bigcap_{p} p A$.

If $A$ is any ring, we introduce the ring $A^{*}$ in the following way: $A^{*}$ consists of all pairs $\{(a, n)\}, a \in A, n$ any integer. We define

$$
\begin{aligned}
(a, n)+\left(a^{\prime}, n^{\prime}\right) & =\left(a+a^{\prime}, n+n^{\prime}\right), \\
(a, n)\left(a^{\prime}, n^{\prime}\right) & =\left(a a^{\prime}+n a^{\prime}+n^{\prime} a, n n^{\prime}\right) .
\end{aligned}
$$

${ }^{3}$ For the definition of the tensor product, see H. Whitney, Tensor products of abelian groups, Duke Math. J. vol. 4 (1938) pp. 495-528. 
It is clear that $(0,1)$ is the unit element of $A^{*}$. If $\mathfrak{M}^{*}$ is an $A^{*}$-module, it is immediately an $A$-module. Conversely, if $\mathfrak{M}$ is an $A$-module, $\mathfrak{M}$ can be made into an $A^{*}$-module by writing $(0,1) x=x$, for all $x \in \mathfrak{M}$. If $\mathfrak{A}^{*}$ and $\mathfrak{A}$ are the annihilators in $A^{*}$ and $A$, it is clear that $\mathfrak{A}^{*} \supset(\mathfrak{A}, 0)$. We have then that the extension radical of $A^{*}$ contains $(\Re, 0)$. But $A^{*}$ has a unit element, so that its extension radical is $T^{*} \cap \bigcap_{p} p A^{*}$. However, $T^{*}=(T, 0)$ and $\bigcap_{p} p A^{*}=\left(\bigcap_{p} p A, 0\right)$, so that the extension radical of $A^{*}$ is equal to $\left(T \cap \bigcap_{p} p A, 0\right)$. This together with the preceding remark gives $\Re \subset T \cap \cap_{p} p A$, so that $\Re=T \cap \cap_{p} p A$, concluding the proof of the theorem.

The condition that the extension radical reduces to $\{0\}$ is equivalent to the statement that there are no elements of order $p^{2}$ in the additive group of $A$, for all primes $p .{ }^{4}$ For, suppose first that $T \cap \cap_{p} p A=\{0\}$, and $p^{2} a=0$ for some $p$ and some $a$. Then for any prime $q$, we can write $p a=q r a$, where $r$ is any solution of the congruence $p \equiv q r\left(\bmod p^{2}\right)$ (that such an $r$ exists follows from the fact that $q$ and $p^{2}$ are relatively prime when $q \neq p$; in the remaining case $r=1$ will suffice). $p a$ is then in the extension radical, so that $p a=0$. Conversely, suppose that $a \in T \cap \bigcap_{p} p A$. Let $n$ be the order of $a$, and let $p$ be any prime factor of $n$; we have then $p m a=0$, where $p m$ is the small est positive integer with this property. Since $a$ is in the extension radical, we have, for some $b, a=p b$. But then $p^{2} m b=0$, so that, if there are no elements of order $p^{2}$, we have already $p m b=0$ or $m a=0$, contradicting the supposition that $p m$ is the order of $a$. Thus $a=0$, and hence the extension radical is $\{0\}$.

Combining this result with Theorems I and II, we see that we have already proved the following theorem.

THEOREM III. Let $G$ be an abelian group, and let $\Gamma$ be the set of all rings having $G$ as underlying additive group. Then, a necessary and sufficient condition that each element of $\Gamma$ has a semi-simple extension is that $G$ have no element of order $p^{2}$ for any prime number $p$.

Princeton University

4 I am indebted to the referee for calling my attention to this fact. 